\title{
Celiac disease in the adult
}

Celiac disease $(\mathrm{CD})$ is an autoimmune systemic condition -the only one with a well-established origin- resulting from permanent gluten intolerance, which primarily involves the gut. It is characterized by the presence of chronic mucosal inflammation in the small bowel, which may be associated with highly variable clinical manifestations. It may develop at any age, both during childhood and adolescence, and is relatively common in th adult; it is increasingly diagnosed even in elderly patients (up to $20 \%$ of patients are older than 60 years at the time of diagnosis).

The causal agent is gluten, a protein preset in cereal -mainly wheat, barley, rye and oats, but not corn- flour. It affects predisposed individuals whose most susceptible genetic features are identified in the HLA-II histocompatibility system of human leukocytes, and whose most common markers include HLA-DQ2 (90\%) and HLA-DQ8 (5-10\%). However, such genetic markers are a necessary but insufficient condition, since with a non-negligible frequency (5-10\%) celiac patients are encountered who are DQ2- and DQ8-negative, and thus other still not well-established genotypes may exist that probably correspond to the class-I HLA system, including MICA and MICB.

Most affected individuals show sustained clinical remission when strictly on a gluten-free diet (GFD), which must be kept indefinitely for life as a result of this illness having genetic grounds.

Until recently, $\mathrm{CD}$ was considered a rare disease, but is known nowadays to be universally distributed, to involve all races, and to be one of the most common genetic diseases with a mean prevalence of $1-2 \%$ in the general population, being clearly subestimated and subdiagnosed worldwide. This would represent an estimate of 3 million people affected by CD in Europe, and around 450,000 in the whole of Spain (only around 45,000 are currently diagnosed).

Selected ethnic groups may have a prevalence lower than that suggested for the Caucasian race but not far from them, as the worldwide distribution of CD is pretty homogeneous, the only exception extant being the Saharan population with a mean estimate of $10 \%$, the highest heard of thus far (1-6).

Most common gastrointestinal manifestations include frequent abdominal pain and distension episodes, prolonged digestion, and altered bowel habit (diarrhea and/or constipation) in association with trace element, vitamin and mineral deficiencies, this being the reason that it is often accompanied by anemia, osteoporosis, and other extra-intestinal manifestations.

While diarrhea was almost considered a necessary symptom, this is not the case in the adult, and up to $50 \%$ of patients have constipation predominantly, which on many an occasion becomes refractory to all sorts of therapy used. It is also fit to remember that up to $30 \%$ of celiac patients have obvious overweight at diagnosis. 
Among serologic tests, the measurement of anti-gliadin IgA antibodies (AGA) has completely fallen into disuse, and justifiably abandoned, as sensitivity and specificity are very low (around 50\%).

In its stead IgA antibodies against tissue transglutaminase (TTG) are used, which are measured using ELISA, exhibit high sensitivity and specificity (80-95\%), and are to be preferred versus anti-endomisium antibodies (EMA), as these are tested using immunofluorescence and explore the same substract, namely tissue transglutaminase type 2 (TTG), thus having the same diagnostic reliability; as their measurement is labour-intensive and costly, only the former are measured in daily clinical practice.

The findings of duodenal bipsy must be interpreted in detail by a pathologist experienced and interested in this condition and according to Marsh's criteria (modified), which stratify this disease in 4 types or stages. Biopsy samples are usually collected during endoscopy, and 4-6 well-oriented samples should be submitted, as these are often irregular, patchy lesions.

CD may be diagnosed with a normal duodenal biopsy (stage 0), or just an increased percentage of intraepithelial lymphocytes above $30 \%$ (stage 1), or crypt hyperplasia and presence of inflammatory infiltrates in the submucosa (stage 2), all of them with well-preserved villi. It is not until stage 3 that villi atrophy develops, which is substratified in three categories: mild (A), moderate (B), and total or subtotal (C). Finally, stage 4 or hyperplasia is now only rarely seen.

Routine use of Marsh's classification in the study of duodenal biopsies is of relevant help when recognizing occult, doubtful cases, and must be always the case when performing a diagnostic gastroscopy in patients belonging to so-called "atrisk groups" regarding $\mathrm{CD}$, including those with chronic diarrhea, refractory ferropenic anemia, and so on (7-13).

Duodenal biopsy is still considered by most authors the "gold standard" in the diagnostic process, albeit its usefulness in the adult is presently controversial given the high diagnostic sensitivity of serologic methods currently available.

The wide spectrum of this disease must be recognized and related to the reported range of laboratory and histopathologic disturbances. It is with a combination of clinical, laboratory, and duodenal biopsy findings, plus patient response to GFD, that a diagnosis is most easily reached.

For diagnosis confirmation in uncertain cases several potential strategies may be agreed upon according to patient and family preferences. Amongst them we shall quote the following: a) periodically repeat serology and lab tests; b) perform a full family exploration; c) perform a new endoscopy with biopsy collection (less accepted); and d) try GFD for 6 months, monitoring clinical response as well as serologic and laboratory changes.

All patients newly diagnosed with CD should prompt a systematic screening of all first-degree relatives, as these have a high risk for CD even in a subclinical form, with a prevalence around $12 \%$; second-degree relatives also are more involved when compared to the general population. Monozygotic twins are highly concordant, around $75 \%$, whereas the frequency encountered in dizygotic twins does not differ from that seen in first-degree relatives, which reveals the presence of a strong genetic influence in this condition $(14,15)$.

Patients with type- 1 diabetes mellitus have a high frequency of accompanying $\mathrm{CD}$, the percentage oscillating between 3 to $8 \%$. Children with Down's syndrome have this disease even more frequently $-5-12 \%$. CD is also associated with other genetic disturbances such as Turner's syndrome, Williams' syndrome, IgA deficiency, and a number of autoimmune conditions. 
The small intestine has a considerable functional reserve, which accounts for the fact that many patients have few or no gastrointestinal symptoms, and most exhibit no malabsorption.

Clinical presentation is highly variable and depends upon various factors, including patient age, gluten susceptibility extent, and amount of cereal flour ingested with the diet, as well as othe factors unknown so far.

$\mathrm{CD}$ exhibits strong clinical variability among patients involved, with a very wide spectrum of manifestations. The classic presentation is characterized by frequent diarrhea and steatorrhea episodes, abdominal distension, limb edemas, and severe asthenia. The latter form of presentation is most uncommon in the adult.

In contrast, the usual form of presentation is oligosymptomatic, with atypical complaints such as constipation, predominantly extra-digestive manifestations or absolute lack of symptoms, the so-called "silent forms". It is clear from a number of recently performed epidemiologic studies that $\mathrm{CD}$ may be 10 times more common than presently perceived.

Disease presentation may be related to an extrinsic episode of acute gastroenteritis (AGE), a trip abroad (particularly tropical countries), stress, or surgery.

Patients with functional dyspepsia or irritable bowel syndrome meeting Roma II clinical criteria have a potential for being celiac in $10-20 \%$ of cases.

The presence of herpetiform dermatitis-like skin lesions, characterized by the presence of vesicles, scabs, and localized fissures on the extensor surfaces of limbs, but also on the palms and soles as well as the scalp, associated with severe pruritus, is seen in 1 of 4 celiac patients $(25 \%)$ and are so typical that they are designated "skin CD" and considered a celiac patient's "calling card" (14-18).

On occasion extra-intestinal manifestations are very prominent and varied in nature -neurological (headaches, ataxia, multiple sclerosis, narcolepsy, etc.), rheumatic (rheumatoid arthritis, ankylosing spondylitis, fibromyalgia), hematologic (thrombocytopenic purpura), etc. (19).

CD impairs perceived health status in affected individuals, which improves up to normal levels when on a chronic GFD as Casellas et al. revealed after an HRQoL study in 60 celiac patients (20).

CD therapy consists only of a strict gluten-free diet that must be continually kept. Gluten-free foods are usually more costly (5-fold on average) than normal foods, and entail difficulties regarding strict adherence, which notably restricts social life for involved patients. A therapy trial using a gluten-free diet may be attempted in uncertain cases -patients demonstrating a positive clinical and laboratory response are very likely to have concealed $\mathrm{CD}$.

Many patients report a fast improvement when on GFD, which develops as early as within 48 hours. Notwithstanding, response is not so early in a variable percentage of cases (20-40\%) and may take several weeks in becoming apparent, while labconfirmed objective results are better seen after 2-4 months $(21,22)$.

Products containing wheat, barley or rye must be fully excluded from the diet. In fact, a complete exclusion of gluten is very difficult to attain and then keep, as wheat is used as a thickening agent in many manufactured products, including canned food, sausage, foie-gras, chocolate, ice-cream, etc.

The only gluten-free cereal is corn, which is therefore allowed in the celiac patient's diet whether raw or roasted, in salads, etc. Corn flour, if pure (100\%) regarding preparation, processing and manufacture, may be used for bread, cookies, baby foods, and sweets, which may render a celiac patient's diet more bearable. 
Dairy products must be avoided in non-treated patients, as secondary lactase deficiency is often associated with celiac disease. After 1-2 months on gluten-free diet milk derivatives may be gradually reintroduced as far as the patient has no complaints following ingestion, since lactose intolerance is secondary to gluten and usually regresses within 3 months with strict gluten-free diet adherence. On the other hand, dietary calcium and proteins are essential to correct existing mineral deficiencies, given the high prevalence of osteoporosis seen in celiac patients.

Beer must be completely avoided, even alcohol-free brands, as its manufacture involves the fermentation of various gluten-containing cereals, including barley and rye, and the same can be said of whisky based on malt distillation procedures.

Approximately $70 \%$ of patients experience symptom improvement after 2 weeks on GFD. The rapidity and extent of histologic regression are unpredictable, but there is invariably a delay versus clinical improvement, and may not be apparent in repeat biopsies until after three to six months. While histologic findings usually regress in children, one half of adults only achieve a partial histologic resolution. When there is severe ferropenic anemia the administration of iron preparations through the endovenous or intramuscular route is recommended fo a few months in order to shorten recovery time.

The most common cause of failed response is incomplete diet adherence, which may be intentional or unintentional; hence, support groups such as national or regional celiac disease associations are essential for adequate patient education regarding gluten-free foods, suggesting safe products and brands.

As Dr. Casellas et al. point out in the present issue of our journal, it is essential that patients be well motivated regarding strict GFD adherence, and they should also know for certain which foods and manufactured products may be safely used, and which should be avoided (23).

Adequate disease control may be obtained through appropiate coordination and cooperation among professionals involved, particularly specialists and primary care physicians, and based on clear, objective information from regional celiac sprue associations and sources such as specialized dietary services or even the world-wide web.

Health authorities should help this kind of patients by lowering gluten-free food prices, providing this sort of diet in school dining rooms, subsidizing "celiac menus" in restaurants, and lending financial support to families in need.

Several issues may be of help, as listed in the following decalogue:

1. Have a better understanding of $\mathrm{CD}$, think of it as a very common disease (1-2\% general population), and consider celiac sprue in the differential diagnosis of multiple gastrointestinal or otherwise conditions.

2. Obtain a careful clinical history since childhood, looking for a relation of periodic complaints to food ingestion, exploring the family's history of celiac disease and associated immunoallergic conditions such as recurrent rhinitis, pharyngoamigdalitis, otitis, sinusitis, bronchial asthma, thyroiditis, and various dermatitides.

3. Systematic searching in patients with iron deficiency and/or chronic ferropenic anemia refractory to replacement therapy using oral iron preparations, and routinely collecting duodenal biopsies during upper gastrointestinal endoscopies.

4. In the presence of sustained hypertransaminasemia, in the absence of a history of liver disease, and when viral markers are negative, screening for $\mathrm{CD}$, which is associated to the former conditions in around $10 \%$ of cases.

5. If endocrine disturbances such as hypo- or hyperthyroidism, or simply positive anti-microsomal antibodies with normal thyroideal function are present, and in 
the presence also of associated diabetes mellitus, particularly in insulin-dependent cases, consider a potential CD.

6. Consider that screening serology, particularly the measurement of anti-transglutaminase antibodies, is often negative in adult patients.

7. Remember that duodenal biopsy is usually normal or has minimal changes, and that an experienced pathologist deeply interested in the diagnosis of CD and routinely using Marsh's classification in his or her reports is needed.

8. Bear in mind that being DQ2 ( + ) is a necessary but insufficient condition, and that increasingly more cases are being diagnosed in DQ2 (-) individuals.

9. In doubtful cases gluten-free diet may be suggested for a minimum of 6 months, and then patient clinical and laboratory response is observed ("ex-iuvantibus" diagnosis).

10. Finally, all gastroenterology departments should include monographic "Small Bowel Units" for the protocolized study of these patients, and said Units should be fitted with modern functional techniques such as capsule endoscopy and duble-balloon entroscopy.

\section{Rodrigo Sáez}

\section{Service of Digestive Diseases. Central University Hospital of Asturias.} School of Medicine. Oviedo, Asturias. Spain

\section{References}

1. James WJ, Scout BB. Celiac disease: the cause of the various associated disorders? Eur J Gastroenterol Hepatol 2001; 13: 1119-21.

2. Catassi C, Ratsch IM, Fabiani E, Rossini M, Bordicchia F, Candela F, et al. Coeliac disease in the year 2000: exploring the iceberg. Lancet 1994; 343: 200-3.

3. Green PH, Jabri B. Coeliac disease. Lancet 2003; 362: 383-91.

4. Lo W, Sano K, Lebwohl B, Diamond B, Green PH. Changing presentation of celiac disease. Dig Dis Sci 2003; 48: 395-8.

5. Fergusson A, Arranz E, O`Mahony S. Clinical and pathological spectrum of coeliac disease-active, silent, latent, potential. Gut 1993; 34: 150-1.

6. Fasano A, Berti I, Gerarduzzi T, Not T, Colletti RB, Drago S, et al. Prevalence of celiac disease in at-risk and not-at-risk groups in the United States: a large multicenter study. Arch Intern Med 2003; 163: 286-92.

7. Tommasini A, Not T, Kiren V, Baldas V, Santon D, Trevisiol C, et al. Mass screening for celiac disease using antihuman transglutaminase antibody assay. Arch Dis Child 2004; 89: 512-5.

8. Riestra S, Fernandez E, Rodrigo L, Garcia S, Ocio G. Prevalence of coeliac disease in the general population in northern Spain. Strategies of serologic screening. Scand J Gastroenterol 2000; 35: 398-402.

9. Kakar S, Nehra V, Murray JA, Dayharsh GA, Burgart LJ. Significance of intraepithelial lymphocytosis in small bowel biopsy samples with normal mucosa architecture. Am J Gastroenterol 2003; 98: 2027-33.

10. Jarvinen TT, Collin P, Rasmussen M, Kyronpalo S, Maki M, Partanen J, et al. Villous tip intraepithelial lymphocytes as markers of early-stage celiac disease. Scand J Gastroenterol 2004; 39: 428-33.

11. López-Vázquez A, Rodrigo L, Fuentes D, Riestra S, Bousoño C, García-Fernández S, et al. MHC class I chain related gene A (MICA) modulates the development of coeliac disease in patients with th high risk heterodimer DQA $1 * 0501 / \mathrm{DQB} 1 * 0201$. Gut 2002; 50: 336-40.

12. Hogberg L, Falth-Magnusson K, Grodzinsky E, Stenhammar L. Familial prevalence of coeliac disease: a twenty-year follow-up study. Scand J Gastroenterol 2003; 38: 61-5.

13. Fernández E, Riestra S, Rodrigo L, Blanco C, López-Vázquez A, Fuentes D, et al. Comparison of six human anti-transglutaminase ELISA-tests in the diagnosis of celiac disease in the Saharawi population. World J Gastroenterol 2005; 11: 3762-6.

14. Rodrigo L, Riestra S, Fuentes D, González S, López Vázquez A, López Larrea C. Diversas formas de presentación clínica de la enfermedad celíaca en la misma familia. Rev Esp Enferm Dig 2004; 96: 612-9.

15. Cadahía V, Rodrigo L, Fuentes D, Riestra S, de Francisco R, Fernández M. Enfermedad celíaca (EC), colitis ulcerosa (CU) y colangitis esclerosante primaria (CEP) asociadas en el mismo paciente: Estudio familiar. Rev Esp Enferm Dig 2005; 97: 907-13

16. Antonioli DA. Celiac disease: A progress report. Modern Pathol 2003; 16: 342-6. 
17. Tursi A, Brandimarte G. The symptomatic and histologic response to a gluten-free diet in patients with borderline enteropathy. J Clin Gastroenterol 2003; 36: 13-7.

18. Ciclitira PJ, King AL, Fraser JS. AGA technical review on celiac sprue. Gastroenterology 2001; 120: 152640.

19. González S, Rodrigo L, Salas Puig J, Astudillo A, Fuentes D, López Vázquez A, et al. Ataxia mioclónica progresiva asociada con anticuerpos frente a las células de Purkinje en paciente con enfermedad celíaca. Rev Esp Enferm Dig 2005; 97: 917-26.

20. Casellas F, López Vivancos J, Malagelada JR. Percepción del estado de salud en la enfermedad celíaca. Rev Esp Enferm Dig 2005; 97: 794-804.

21. Wong RC, Steele RH, Reeves GE, Wilson RJ, Pink A, Adelstein S. Antibody and genetic testing in celiac disease. Pathology 2003; 35: 285-304.

22. Dewar DH, Ciclitira J. Clinical features of celiac disease. Gastroenterology 2005; 128: S19-S24.

23. Casellas F, López Vivancos J, Malagelada JR. Epidemiología actual y accesibilidad al seguimiento de la dieta de la enfermedad celíaca del adulto. Rev Esp Enferm Dig 2006; 98 (6): 408-19. 